\title{
Ueber eine Methode zur Bestimmung der Constitution von gesättigten und ungesättigten Halogenderivaten, resp. Kohlenwasserstoffen;
}

ron

\section{Mejer Wildermann.}

Während die Halogenderivate des Methans, Aethans, Propans zahlreich dargestellt und ihrer Constitution nach bestimmt worden sind, ist dies bei den höheren Kohlenwasserstoffen, schon von den Butanen an, nicht mehr der Fall; hier sind nur noch die Halogenderivate mit ein und zwei Atomen Halogen ihrer Constitution nach bestimmt worden, die mit höherem Halogengehalt, sind nur ihrer empirischen Zusammensetzung, nicht ihrer Constitution nach, bekannt. Noch mehr ist dies der Fall mit den ungesättigten Verbindungen: die ungesättigten Kohlenwasserstoffe, resp. ungesättigten Halogenderivate mit einer Doppelbindung, oder mit einer dreifachen Bindung sind ihrer Constitution nach ermittelt worden, dagegen ist die Constitution von ungesättigten Kohlenwasserstoffen, resp. ungesättigten Halogenderivaten mit mehreren doppelten (schon von zwei an) oder dreifachen Bindungen nur in seltenen Fällen ermittelt worden. Der Grund davon liegt bekanntlich darin, dass die wichtigsten uns zur Verfügung stehenden Methoden zur Constitutionsbestimmung, wie die Oxydation von ungesättigten Kohlenwasserstoffen, resp. von ungesättigten Halogenderivaten, sowie die Einführung von Hydroxyl-Carboxylgruppen an Stelle der Halogene u. s. w. nicht ausreichen, um die Constitution solcher Verbindungen zu bestimmen. Bei der grossen Wichtigkeit, welche die Kenntniss der Constitution in der organischen Chemie für uns hat, scheint es mir von Interesse zu sein, eine Methode der Constitutionsbestimmung anzugeben, welche dort noch gute Dienste leisten kann, wo die anderen, wichtigsten uns zur Verfügung stehenden Methoden der Constitutionsbestimmung schon versagen, und mittelst welcher man auch die Constitution von ungesättigten Verbindungen mit mehreren doppelten und dreifachen Bindungen, sowie die Constitution von Halogenderivaten mit höherem Halogengehalt bestimmen kann. 
Die Grundlage der vorliegenden Methode bildet ein längst bekanntes mathematisches Princip; sie besteht darin, dass man die Zahl der für die Constitution der betreffenden gesättigten oder ungesättigten Verbindung theoretisch möglichen Combinationen bis auf eine theoretisch einzig mögliche zu reduciren sucht; die vorliegende Methode selbst besteht darin, dass sie diese Reduction der Combinationen durch successive Anlagerung von Brom bisozur Sättigung und Maximalabspaltung von Bromwasserstoff direct und indirect durchführt.

Die systematische Reduction der theoretisch-möglichen Combinationen gelingt auf folgende Weise:

Haben wir z. B. die Constitution eines gesättigten oder ungesättigten Halogenderivates, resp. Kohlenwasserstoffs zu bestimmen, welches aus einer ungesättigten Verbindung durch Anlagerung von ein, zwei, drei u. s. w. Molekülen Brom oder aus einer gesättigten Verbindung durch Abspaltung von ein, zwei, drei u. s. w. Molekülen Bromwasserstoff entstanden ist, so berechnen wir zuerst, welche Combinationen für die Constitution der betreffenden Verbindung möglich sind. Angenommen, wir untersuchen die ungesättigte Verbindung, die durch Abspaltung von drei Mol. Bromwasserstoff aus einer Verbindung von bestimmter Constitution entstanden ist, und die theoretischen Combinationen für die betreffende Verbindung seien $A, B, C, D, E, F, G$. Zuerst suchen wir auf Grund der uns zur Zeit zu Gebote stehenden physikalischen und chemischen Hilfsmittel die Zahl der Combinationen direct zu reduciren. Unterscheiden sich z. B. die Combinationen $A, B, C, D$ von den Combinationen $E, F, G$ durch die Art der Bindungen, d. h. enthalten z. B. die ersteren drei doppelte Bindungen, die letzteren eine doppelte und eine dreifache, so können wir die Combinationen $A, B, C, D$ von den Combinationen $\boldsymbol{E}, \boldsymbol{F}, \boldsymbol{G}$ auf Grund der Verbrennungswärme mit Sicherheit unterseheiden, wenn unsere Verbindung (eine offene Kette) ein ungesättigter Kohlenwasserstoff oder ein ungesättigtes Halogenderivat mit niederem Halogengehalt, mit ein oder zwei Halogenen, ist. ${ }^{1}$ )

1) Vergl. Thomsen, Therm. Unters. 4; die Kritik von Brühl, dies. Journ. [2] 35, 182-185; die Antwort Thomsen's, Z. phys. Ch. 1, Heft 7, 
Ergiebt sich, dass die noch theoretisch möglichen Combinationen $E, F, G$ sind, und unterscheidet sich $E$ von $F, G$ dadurch, dass das erstere ammoniakalisches Kupferchlorür fällt, die anderen nicht, so untersuchen wir unsere ungesättigte Verbindung auf ihr Verhalten gegen ammoniakalisches Kupferchlorür u. s. w.

Kann man die noch als theoretisch möglich übrig gebliebenen Combinationen $A, B, C, D$ oder $F, G$ auf Grund der uns zu Gehote stehenden chemischen und physikalischen Hilfsmittel nicht mehr direct reduciren, so setzt man die weitere Ausschliessung von Combinationen indirect fort. Der Möglichkeit, durch eine indirecte Untersuchung die Zabl der Combinationen weiter $\mathrm{zu}$ reduciren, ist es $\mathrm{zu}$ verdanken, dass vorliegende Methode anwendbar wird. Diese indirecte Untersuchung besteht darin, dass man die betreffende ungesättigte Verbindung in seinem Halogenadditionsprodukte oder in dem aus dem Halogenadditionsprodukte entstandenen neuen ungesättigten Halogenderivat, resp. ungesättigten Kohlenwasserstoff weiter untersucht.

Angenommen, es sind noch die Combinationen $A, B, C, D$ (mit drei doppelten Bindungen) als theoretisch möglich übriggeblieben, derart, dass wir sie direct nicht weiter reduciren können. Wir addiren dann an die betreffende Verbindung Brom bis zu voller Sättigung und bewirken das Maximum der Bromwasserstoffabspaltungen. Da die theoretischen $A, B, C, D$ der Constitution nach verschieden sind, so kann es sehr gut vorkommen, dass sie Halogenadditionsprodukte geben werden, welche der Constitution nach auch verschieden sind und nicht dieselbe Zahl von Mol. Bromwasserstoff abspalten können; und aus der Zah! der Bromwasserstoffatome, die unser Halogenderivat in der Wirklichkeit abspalten kann, lässt sich daher oft der Rückschluss ziehen, welche dieser theoretischen Halogenadditionsprodukte unserem Halogenderivat noch zukommen können. Haben wir auf diese Weise eine Reihe von Combinationen aus der

S. 369-375; Z. phys. Chem., 7, Heft 1, S. 55-70; Brühl, Ber. 1891, Heft 4, S. $650-656$ u. s. w. 
Zahlder für das Halogenadditionsprodukt theoretisch-möglichen Combinationen ausgeschlossen, so ist dadurch gleichzeitig indirect eine Reihe von Combinationen aus der Zahl der theoretischen Combinationen, welche unserem ungesättigten Halogenderivat, a us welchem das Halogeuadditionsprodukt entstanden ist, zukommen können, auch ausgeschlossen. Können dagegen die theoretischen Halogenadditionsprodukte dieselbe Zahl von Mol. Bromwasserstoff abspalten, so müssen doch, mit wenigen Ausnahmen, die aus verschieden constituirten theoretischen $A, B, C, D$ nach Anlagerung von Brom bis zur Sättigung und Maximalabspaltung von Bromwasserstoff entstandenen neuen ungesättigten Verbindungen theoretisch der Constitution nach auch verschieden sein. Zuerst berechnen wir die Combinationen, die aus jedem der Combinationen $A, B, C, D$ nach Anlagerung ron Brom und Abspaltung von Bromwasserstoff entstehen können. Angenommen, aus $A$ können theoretisch $a^{\prime}, a^{\prime \prime}, a^{\prime \prime \prime}$, aus $B b^{\prime}$, aus $C c^{\prime}, c^{\prime \prime}, c^{\prime \prime \prime}$, aus $D d^{\prime}$ und $d^{\prime \prime}$ entstehen. Wir suchen jetzt auf Grund der uns zur Verfügung stehenden chemischen und physikalischen Hilfsmittel die Combinationen $a^{\prime}, a^{\prime \prime}, a^{\prime \prime \prime}, b^{\prime}, c^{\prime}, c^{\prime \prime}, c^{\prime \prime \prime}, d^{\prime}, d^{\prime \prime}$ auf ein Minimum direct zu reduciren. Die nach dieser Reduction für die neue ungesättigte Verbindung noch als theoretisch möglich übrig gebliebenen Combinationen dürfen aber nicht aus allen Combinationen $A, B, C, D$ entstanden sein. Angenommen, dass die directe Untersuchung der neuen ungesättigten Verbindung gezeigt hat, dass für diese letatere nur noch die Combinationen $a^{\prime}, a^{\prime \prime}, c^{\prime}, e^{\prime \prime}, d^{\prime}, d^{\prime \prime}$ theoretisch möglich sind, so wird dadurch aus den theoretisch-möglichen Combinationen $A, B, C, D$ für die erste ungesättigte Verbindung indirect die theoretische Combination $B$ ausgeschlossen. Die directe Reduction der Combinationen für die neue ungesättigte Verbindung hat uns somit ermöglicht, indirect die Zahl der theoretischen Combinationen für die erste ungesättigte Verbindung weiter zu reduciren. Ebenso, wie wir die Combinationen $A, B, C, D$ indirect durch Anlagerung von Brom und Abspaltung von Bromwasserstoff auf $A, C, D$ reducirt haben, werden wir weiter die Combinationen $a^{\prime}, a^{\prime \prime \prime}, c^{\prime}, c^{\prime \prime}$, $d^{\prime}, d^{\prime \prime}$ durch eine neue Anlagerung von Brom und Abspaltung 
von Bromwasserstoff auf $a^{\prime}, d^{\prime}, d^{\prime \prime}$ reduciren können; das bedeutet aber in $z$ weiter Linie indirect die Combinationen $A, C, D$ auf $A$ und $D$ zu reduciren. Auf diese Weise können wir, ausgehend von einem und demselben Körper, durch successive Anlagerung von Brom bis zur Sättigung und Maximalabspaltung von Bromwasserstoff die Zahl der für die betreffende ungesättigte Verbindung theoretisch möglichen Combinationen bis auf ein Minimum reduciren.

Wir erhalten auf diese Weise, ausgehend von einem bestimmten Körper, eine Reihe von gesättigten und ungesättigten Verbindungen, welche sich successiv aus einander durch Anlagerung von Brom bis zur Sättigung und Maximalabspaltung von Bromwasserstoff gebildet haben. Aus dem innigen $\mathrm{Zu}$ sammenhange, in welchem sich diese Verbindungen befinden, folgt, dass wir nicht nur die gesuchte Constitution der ersten Verbindung, von welcher wir ausgingen, bestimmen werden, sondern gleichzeitig auch die Constitution der meisten anderen Glieder dieser Reihe bei dieser Constitutionsbestimmung des ersten Gliedes erkennen werden.

Es kann aber vorkommen, dass während der successiven Anlagerung von Brom und Abspaltung von Bromwasserstoff Umlagerungen, Zersetzungen oder andere Hindernisse entstanden sind, so dass es nothwendig oder wünschenswerth wäre, die Reduction der Combinationen durch die successive Untersuchung eines Körpers nur bis zu einem gewissen Stadium durchzutühren, die weitere Reduction der Combinationen aber auf irgend eine andere Art fortzusetzen. Eine solche zweite Art Reduction der Combinationen ist in der That auch möglich.

Angenommen, aus irgend welchen Gründen könnten wir die Combinationen $A, B, C, D, E, F, G$ nur bis auf $A$ und $C$ reduciren. Die theoretischen Combinationen $A$ und $C$, welche (X-3BrH) sind, unterschieden sich aber dadurch, dass die Stellung der einen, zwei oder sogar aller drei doppelten (resp. dreifachen) Bindungen in ihnen eine verschiedene ist. Angenommen, die theoretische Combination $A$ enthielte die Doppelbindungen in $c_{-}, \gamma-\gamma$-Stellung, die theoretische Combination $C$ dagegen in $\beta, \delta, \varepsilon$-Stellung, so kann $(\mathrm{X}-\mathbf{1} \mathrm{BrH})$, wenn unser Körper $(\mathrm{X}-3 \mathrm{~B} \cdot \mathrm{H}) \&$ ist, die Doppelbindung nur in einer der 
Stellungen $\alpha, \gamma, \delta$ enthalten; und ( $\mathrm{X}-1 \mathrm{BrH})$ kann die Doppelbindung nur in einer der Stellungen $\beta, \delta, \varepsilon$ enthalten, wenn $(\mathrm{X}-3 \mathrm{BrH}) C$ ist. Ergiebt sich nun in der Wirklichkeit, dass in $(\mathrm{X}-1 \mathrm{BrH})$ die Doppelbindung in der $\alpha$-oder $\gamma$-Stellung sich befindet, so kann die Constitution des $(X-3 \mathrm{BrH})$ nur $A$ und nicht $C$ sein; ergiebt sich, dass im $(\mathrm{X}-1 \mathrm{BrH})$ die Doppelbindung in der $\beta$ - oder $\gamma$-Stellung sich befindet, so kann ( $\mathrm{X}-3 \mathrm{BrH})$ nur $C$ und nicht $A$ sein; dagegen wird (X-3 $\mathrm{BrH})$ immer noch $A$ und $C$ sein können, wenn $(\mathrm{X}-1 \mathrm{BrH})$ die Doppelbindung in $\delta$.Stellung enthalten wird. In diesem letzten ungünstigen Falle werden wir weiter noch $(\mathrm{X}-\mathbf{2} \mathbf{B r H})$ untersuchen, um die Constitution des $(\mathrm{X}-3 \mathrm{BrH})$ näher zu bestimmen. $(\mathrm{X}-2 \mathrm{BrH}) \mathrm{kann} \alpha$ und $\gamma, \alpha$ und $\delta, \gamma$ und $\delta$ sein, wenn $(\mathrm{X}-3 \mathrm{BrH}) A$ ist, und $\beta$ und $\delta, \delta$ und $\varepsilon$ sein, wenn $(\mathrm{X}-3 \mathrm{BrH}) D$ ist. Unter allen Umständen müssen die Combinationen $A$ und $B$ für $(\mathrm{X}-3 \mathrm{BrH})$ auf $A$ oder $B$ reducirt werden, wenn wir einmal die Constitution des $(\mathrm{X}-2 \mathrm{BrH})$ kennen werden. Aber angenommen, aus irgend welchen Gründen gelang es uns nicht, die Constitution des $(\mathrm{X}-2 \mathrm{BrH})$, $(\mathrm{X}-1 \mathrm{BrH})$ zu erkennen, dann kann es gelingen, beim nächst höher liegenden ungesättigten Gliede der Reihe, $(\mathrm{Y}-\mathrm{aBrH})$ auf Grund der Erkennung der Constitution von ( $\mathrm{Y}-\mathbf{1 B r H})$, $(\mathrm{Y}-2 \mathrm{BrH})$ u. s. w. seine Combinationen [d. h. des $(\mathrm{Y}-\mathbf{a B r H})]$ weiter zu reduciren; das wird aber bedeuten, die Combinationen von $(\mathrm{Y}-\mathrm{aBrH})$ nach der zweiten Art der Reduction direct zu reduciren und die Combinationen von $(\mathrm{X}-3 \mathrm{BrH})$ gleichzeitig indirect zu reduciren. Gelingt diese Art Reduction der Combinationen beim nächst höher liegenden ungesättigten Gliede der Reihe nicht, so kann es beim zweitnächst höher liegendem Gliede der Reihe gelingen; dadurch werden die Combinationen von $(\mathrm{Y}-\mathrm{aBrH})$ indirect reducirt, und die Combinationen von $(\mathrm{X}-3 \mathrm{BrH})$ in zweiter Linie indirect reducirt werden.

Es ist daher klar: 1. Wir werden bei der Benutzung der ersten und zweiten Art von Reduction der Combinationen zu der gesuchten, theoretisch einzig allein möglichen Constitutionsformel für den betreffenden Körper gelangen. 2. Durch die Möglichkeit, diese Reduction auch nach der zweiten Art $\mathrm{zu}$ vollenden, sind wir in Stand gesetzt, die Reduction der Combinationen nach der ersten und zweiten Art so zu leiten, 
dass die von einem gewissen Stadium der successiven Untersuchung desselben Körpers an entstandenen technischen Hindernisse, Umlagerung, Zersetzungen uns doch nicht hindern werden, die gesuchte Constitutionsformel zu finden. 3. Wir können auf mehreren verschiedenen Wegen zu derselben Constitutionsformel für den betreffenden Körper gelangen, und auf diese Weise die erzielten Resultate controlliren; stimmen die nach zwei verschiedenen Wegen erzielten Resultate nicht überein, so sind entweder Umlagerungen vorgekommen oder irgendwo ist bei der Reduction der Combinationen oder bei der technischen Ausführung der Arbeit ein Fehler begangen worden. Vorliegende Methode, im Unterschiede zu den anderen bisherigen, enthält in sich selbst genügende Mittel, um die nach ihr erzielten Resultate zu controlliren und somit die Constitution von $K \ddot{r} r$ pern mit absoluter Sicherheit zu bestimmen.

Die hier besprochene Methode: Reduction der theoretischen Combinationen bis auf eine einzig theoretisch-mögliche wird vor der Hand hauptsächlich zur Bestimmung der Constitution von gesättigten und ungesättigten Kohlenwasserstoffen, resp. Halogenderivaten der Fettreihe benutzt werden können. Es ist aber, vielleicht, die Zeit nicht ferne, wo wir sie auch zur Bestimmung der Constitution von gesättigten und ungesättigten Halogenderivaten anderer Körperklassen, wie z. B. der Fettsäuren, zu benutzen im Stande sein werden, d. h. dass wir sie überall benutzen werden, wo die gesättigten und ungesättigten Halogenderivate der anderen Körperklassen analog, wie bei den Kohlenwasserstoffen der Fettreihe, erhalten werden.

Durch die Berechnung einer Reihe von Beispielen, welche beliebig aus den normalen und Isoverbindungen genommen wurden, werde ich zeigen, dass vorliegende Methode mit sehr gutem Erfolg und in weiten Grenzen zur Bestimmung der Constitution der Körper benutzt werden kann. Da aber die Untersuchung nach der zweiten Art darin besteht, dass wir zur Erkennung der Constitution eines Körpers die Constitution anderer Körper durch successive Anlagerung von Brom und Abspaltung von Bromwasserstoff untersuchen, so scheint es mir von Interesse, zu zeigen, was wir auf Grund der successiven Anlagerung von Brom und Abspaltung von Bromwasser- 
Wildermann: Ueber eine Methode etc.

stoff, ausgehend von einem und demselben Körper, ohne uns zu der zweiten Art der Reduction zu wenden, schon erreichen können.

\section{Beispiel 1.}

Es soll die Constitution des Kohlenwasserstoffs $\mathrm{C}_{7} \mathrm{H}_{12}$ bestimmt werden, welchen man durch Abspaltung von zwei Mol. Bromwasserstoff mittelst alkoholischen Kalis aus $\mathrm{C}_{7} \mathrm{H}_{14} \mathrm{Br}_{2}$ von der Constitution $\mathrm{CH}_{3}$. CHBr. $\mathrm{CHBr}$. $\left(\mathrm{CH}_{2}\right)_{3} \mathrm{CH}_{3}$ erhält.

Das ungesättigte Halogenderivat $\mathrm{C}_{7} \mathrm{H}_{12}$ kann nur eine der folgenden Formeln besitzen:
1. $\mathrm{CH}_{2}=\mathrm{CH} . \mathrm{CH}_{-} \mathrm{CH} . \mathrm{CH}_{2} . \mathrm{CH}_{3}$.
3. $\mathrm{CH}_{3}, \mathrm{CH}_{-} \mathrm{CH}, \mathrm{CH}_{2}, \mathrm{CH}_{2}, \mathrm{CH}_{4}$.
2. $\mathrm{CH}_{2}-\mathrm{C}_{-}^{-} \mathrm{CH} .\left(\mathrm{CH}_{2}\right)_{3} \mathrm{CH}_{3}$.
4. $\mathrm{CH}_{3}, \mathrm{C} \equiv \mathrm{C} \cdot\left(\mathrm{CH}_{2}\right)_{3} \mathrm{CH}_{3}$.

Auf Grund der Verbrennungswärme von $\mathrm{C}_{7} \mathrm{H}_{12}$ kann man sehr gut bestimmen, ob dieses letztere zwei doppelte oder eine dreifache Bindung enthält, denn $\mathrm{C}_{7} \mathrm{H}_{12}$ mit einer dreifachen Bindung wird eine um ca. $13000^{\mathrm{e}}$ höhere Verbrennungswärme besitzen, als $\mathrm{C}_{7} \mathrm{H}_{12}$ mit zwei doppelten Bindungen. Ergiebt sich aus der Verbrennungswärme des $\mathrm{C}_{7} \mathrm{H}_{12}$, dass dieses eine dreifache Bindung enthält, so ist seine Constitution -4 , d. h. $\mathrm{CH}_{3} \cdot \mathrm{C} \equiv \mathrm{C} .\left(\mathrm{CH}_{2}\right)_{3} \cdot \mathrm{CH}_{3}$. Ergiebt sich aber aus der Verbrennungswärme des $\mathrm{C}_{7} \mathrm{H}_{12}$, dass dieses zwei doppelte Bindungen enthält, so sind nur noch die Combinationen 1, 2, 3 möglich. Da wir keine Mittel haben, diese drei Combinationen direct weiter zn reduciren, so setzen wir die weitere Ausschliessung indirect fort: wir addiren theoretisch $\mathrm{zu}$ den Combinationen 1, 2, 3 Brom bis zur Sättigung, bewirken das Maximum der Bromwasserstoffabspaltungen und untersuchen, wie früher, das neu erhaltene ungesättigte Halogenderivat:

1. $\mathrm{CH}_{2}=\mathrm{CH} . \mathrm{CH}-\mathrm{CH} . \mathrm{CH}_{2}, \mathrm{CH}_{2} \cdot \mathrm{CH}_{3}$.

2. $\mathrm{CH}_{2}=\mathrm{C}-\mathrm{CH} .\left(\mathrm{CH}_{2}\right)_{3}, \mathrm{CH}_{3}$.

3. $\mathrm{CH}_{3} \cdot \mathrm{CH}_{-} \mathrm{C}-\mathrm{CH} \cdot \mathrm{CH}_{2}, \mathrm{CH}_{2} \cdot \mathrm{CH}_{3}$.

I. $\mathrm{CH}_{2} \mathrm{Br}$. $\mathrm{CHBr}$. $\mathrm{CHBr}$. $\mathrm{CHBr} \cdot\left(\mathrm{CH}_{2}\right)_{2}, \mathrm{CH}_{3}$.

II. $\mathrm{CH}_{2} \mathrm{Br}, \mathrm{CBr}_{2}, \mathrm{CHBr} .\left(\mathrm{CH}_{2}\right)_{3} \cdot \mathrm{CH}_{3}$.

III. $\mathrm{CH}_{3}, \mathrm{CHBr} \cdot \mathrm{CBr}_{2}, \mathrm{CHBr} \cdot\left(\mathrm{CH}_{2}\right)_{2}, \mathrm{CH}_{3}$.

$1^{\prime} . \mathrm{CH} \equiv \mathrm{C} . \mathrm{C} \equiv \mathrm{C} .\left(\mathrm{CH}_{2}\right)_{2} \cdot \mathrm{CH}_{3}$.

1". $\mathrm{CH} \equiv \mathrm{C} . \mathrm{CH}^{-} \mathrm{C}-\mathrm{CH} . \mathrm{CH}_{2} \cdot \mathrm{CH}_{3}$.

$1^{\prime \prime \prime} . \mathrm{CH}_{2} \mathrm{Br} . \mathrm{C} \equiv \mathrm{C} . \mathrm{CH}_{-}^{-} \mathrm{CH} . \mathrm{CH}_{2}, \mathrm{CH}_{3}$. 


$$
\begin{aligned}
& \text { 1IV, } \mathrm{CH}_{2}-\mathrm{C}_{-} \mathrm{C}_{-}^{-} \mathrm{C}-\mathrm{CH}, \mathrm{CH}_{2}, \mathrm{CH}_{3} \text {. } \\
& \text { 1v. } \mathrm{CHBr}_{-} \mathrm{C}_{-} \mathrm{C}-\mathrm{CH} \text {. }\left(\mathrm{CH}_{2}\right)_{2} \mathrm{CH}_{3} \text {. } \\
& \text { 1vi. } \mathrm{CHBr}_{-} \mathrm{CH} \cdot \mathrm{CH}_{-} \mathrm{CH} \cdot \mathrm{CH}_{2} \cdot \mathrm{CH}_{3} \text {. } \\
& \text { 2'. } \mathrm{CBr} \equiv \mathrm{C}, \mathrm{CH}_{-} \mathrm{CH} .\left(\mathrm{CH}_{2}\right)_{2} \cdot \mathrm{CH}_{3} \text {. } \\
& \text { 2". } \mathrm{CHBr}-\mathrm{C}=\mathrm{C}_{-}^{-} \mathrm{CH} .\left(\mathrm{CH}_{2}\right)_{2} \cdot \mathrm{CH}_{3} \text {. } \\
& \text { 3'. } \mathrm{CH}_{2}-\mathrm{C}_{-} \mathrm{C}=\mathrm{CH} . \mathrm{CH}_{2} \cdot \mathrm{CH}_{3} \text {. }
\end{aligned}
$$

Verlaufen nun in der Wirklichkeit bei unserem Körper $\mathrm{C}_{7} \mathrm{H}_{12} \cdot \mathrm{Br}_{4}$ die Bromwasserstoffabspaltungen in der Weise, dass man nur zu $\mathrm{C}_{7} \mathrm{H}_{9} \mathrm{Br}$ gelangen kann, so kann die Constitution dieses letzteren theoretisch nur $1^{\prime \prime \prime}, 1^{v}, 1^{\mathrm{v}}$, und $2^{\prime}, 2^{\prime \prime}$ sein, und die Combinationen $1^{\prime}, 1^{\prime \prime}, 1^{\text {Iv }}$ und $3^{\prime}$ sind aus der Zahl der theoretisch möglichen ausgeschlossen; erhält man dagegen $\mathrm{C}_{7} \mathrm{H}_{8}$, so sind nur noch die Combinationen $1^{\prime}, 1^{\prime \prime}, 1^{\mathrm{IV}}$ und $3^{\prime}$ möglich, und die übrigen müssen aus der Zahl der möglichen Combinationen ausgeschlossen sein. Wie man sieht, kann man auf Grund der Zahl von Bromwasserstoffatomen, die $\mathrm{C}_{7} \mathrm{H}_{12} . \mathrm{Br}_{4}$ abspaltet, schon die Zahl der Combinationen für $\mathrm{C}_{7} \mathrm{H}_{12}$ von $1,2,3$ auf 1 und 2 oder auf 1 und 3 reduciren.

A) Angenommen, wir sind $\mathrm{zu}_{7} \mathrm{H}_{9} \mathrm{Br}$ gelangt, so sind für dieses letztere theoretisch noch die Combinationen $1^{\prime \prime \prime}, 1^{\mathrm{v}}, 1^{\mathrm{v}}$ und $2^{\prime}, 2^{\prime \prime}$ möglich, für $\mathrm{C}_{7} \mathrm{H}_{12}$ sind nur noch die Combinationen 1 und 2 möglich. Um die Combinationen 1 und 2 für $\mathrm{C}_{7} \mathrm{H}_{12}$ weiter indirect $\mathrm{zu}$ reduciren, müssen wir die Combinationen $1^{\prime \prime \prime}, 1^{\mathbf{v}}, 1^{\mathrm{vI}}$ von $2^{\prime}$, $2^{\prime \prime}$, mit der Formel: $\mathrm{C}_{7} \mathrm{H}_{9} \mathrm{Br}$ unterscheiden können. Wir untersuchen daher $\mathrm{C}_{7} \mathrm{H}_{9} \mathrm{Br}$ :

Ergiebt sich aus der Verbrennungswärme des erhaltenen $\mathrm{C}_{7} \mathrm{H}_{9} \mathrm{Br}$, dass dieses letztere eine doppelte und eine dreifache Bindung enthält, so sind für dasselbe theoretisch die Combinationen $1^{\prime \prime \prime}$ und $2^{\prime}$ möglich, die Combinationen $1^{\mathrm{v}}, 1^{\mathrm{vI}}$ und $2^{\prime \prime}$ müssen aus der Zahl für $\mathrm{C}_{7} \mathrm{H}_{9} \mathrm{Br}$ theoretisch möglichen Combinationen ausgeschlossen werden.

Um nun zu entscheiden, welche dieser Combinationen $1^{\prime \prime \prime}$ und $2^{\prime} \mathrm{C}_{7} \mathrm{H}_{9} \mathrm{Br}$ in Wirklichkeit zukommt, addiren wir Brom bis zur Sättigung und bewirken das Maximum der Bromwasserstoffabspaltungen:

$$
\begin{array}{cc}
1^{\prime \prime \prime} . & \mathrm{CH}_{2} \mathrm{Br} . \mathrm{C} \equiv \mathrm{C} \cdot \mathrm{CH}_{-}^{-} \mathrm{CH} \cdot \mathrm{CH}_{2} \cdot \mathrm{CH}_{3} . \\
2^{\prime} . & \mathrm{CBr} \equiv \mathrm{C} \cdot \mathrm{CH}_{-} \mathrm{CH} \cdot\left(\mathrm{CH}_{2}\right)_{2} \cdot \mathrm{CH}_{3} .
\end{array}
$$


Wildermann: Ueber eine Methode etc.

I'. $\mathrm{CH}_{2} \mathrm{Br} \cdot \mathrm{CBr}_{2} \cdot \mathrm{CBr}_{2} \cdot \mathrm{CHBr} \cdot \mathrm{CHBr} \cdot \mathrm{CH}_{2} \cdot \mathrm{CH}_{3}$.

II. $\mathrm{CBr}_{3}, \mathrm{CBr}_{2}, \mathrm{CHBr} . \mathrm{CHBr},\left(\mathrm{CH}_{2}\right)_{2}, \mathrm{CH}_{3}$.

$1^{\mathrm{vI}} \cdot \mathrm{CBr} \equiv \mathrm{C} \cdot \mathrm{CBr}_{2} \cdot \mathrm{C} \equiv \mathrm{O} \cdot \mathrm{CH}_{2} \cdot \mathrm{CH}_{3}$.

1VIII. $\mathrm{CBr} \equiv \mathrm{C} . \mathrm{CBr}_{-} \mathrm{C}=\mathrm{C}=\mathrm{CH} . \mathrm{CH}_{3}$.

2"'. $\mathrm{CBr}_{3} \cdot \mathrm{CBr}_{-}=\mathrm{C}=\mathrm{CH} \cdot \mathrm{CH}_{2} \cdot \mathrm{CH}_{3}$.

2IV. $\mathrm{CBr}_{3} \cdot \mathrm{CBr}_{2} \cdot \mathrm{C} \equiv \mathrm{C} \cdot\left(\mathrm{CH}_{2}\right)_{2} \cdot \mathrm{CH}_{3}$.

Gelangt man zu $\mathrm{O}_{7} \mathrm{H}_{5} \mathrm{Br}_{3}$ oder $\mathrm{zu}_{7} \mathrm{C}_{4} \mathrm{Br}_{2}$, so ist das erstere $1^{\text {VII }}$, das zweite $1^{\text {VIII }}$; die Constitution des $\mathrm{C}_{7} \mathrm{H}_{9} \mathrm{Br} \cdot \mathrm{Br}_{6}$ ist $\mathrm{I}^{\prime}, \mathrm{C}_{7} \mathrm{H}_{9} \mathrm{Br}$ ist $\mathbf{1}^{\prime \prime}, \mathrm{C}_{7} \mathrm{H}_{12} . \mathrm{Br}_{4}$ ist $\mathrm{I}$, und die gesuchte Constitution des $\mathrm{C}_{7} \mathrm{H}_{12}$ ist 1 , d. $\mathrm{h}$.

$\mathrm{CH}_{2}=\mathrm{CH} \cdot \mathrm{CH} \mathrm{CH} . \mathrm{CH}_{2} \cdot \mathrm{CH}_{2} \cdot \mathrm{CH}_{3}$.

Gelangt man dagegen zu $\mathrm{C}_{7} \mathrm{H}_{7} \mathrm{Br}_{5}$ oder $\mathrm{C}_{7} \mathrm{H}_{6} \mathrm{Br}_{4}$, so ist das erstere $2^{\mathrm{lv}}$, das zweite $2^{\prime \prime \prime}, \mathrm{C}_{7} \mathrm{H}_{9} \mathrm{Br} . \mathrm{Br}_{6}$ ist $\mathrm{II}^{\prime}, \mathrm{C}_{7} \mathrm{H}_{9} \mathrm{Br}$ ist $2^{\prime}, \mathrm{C}_{7} \mathrm{H}_{12} . \mathrm{Br}_{4}$ ist II, und die gesuchte Constitution des $\mathrm{C}_{7} \mathrm{H}_{12}$ ist 2 , d. h. $\mathrm{CH}_{2}=\mathrm{CH} \cdot\left(\mathrm{CH}_{2}\right)_{3} \cdot \mathrm{CH}_{3}$.

Ergiebt sich aus der Verbrennungswärme des $\mathrm{C}_{7} \mathrm{H}_{9} \mathrm{Br}$, dass dieses letztere drei doppelte Bindungen enthält, so sind für $\mathrm{C}_{7} \mathrm{H}_{9} \mathrm{Br}$ theoretisch noch die Combinationen $1^{\mathrm{v}}, 1^{\mathrm{vI}}$ und 2 " möglich, die Combinationen $1^{\prime \prime \prime}$ und $2^{\prime}$ sind aus der Zahl der theoretisch möglichen ausgeschlossen. Aus diesen Combinationen sind aber $1^{\mathrm{v}}$ und $2^{\prime \prime}$ identisch. Daraus folgt, dass nur in dem Falle, wenn bei der ersteren Untersuchung sich die Combination $1^{\mathrm{vI}}$ für $\mathrm{C}_{7} \mathrm{H}_{8} \mathrm{Br}$ ergeben wird, die Combinationen 1 und 2 für $\mathrm{C}_{7} \mathrm{H}_{12}$ auf die einzige mögliche Combination 1 reducirt sein wird; wird sich für $\mathrm{C}_{7} \mathrm{H}_{9} \mathrm{Br}$ aber die Constitutionsformel $1^{v}$ (oder $2^{\prime \prime}$ ) ergaben, so werden wir die Combinationen 1 und 2 für $\mathrm{C}_{7} \mathrm{H}_{12}$ hier nicht weiter reduciren können. Wir untersuchen $\mathrm{C}_{7} \mathrm{H}_{9} \mathrm{Br}$ :

$1^{\mathrm{v}}$ (oder 2"). $\mathrm{CHBr}-\mathrm{C}_{-}^{-} \mathrm{C}=\mathrm{CH} \cdot\left(\mathrm{CH}_{2}\right)_{2} \cdot \mathrm{CH}_{3}$. 1vi. $\mathrm{CHBr}_{-} \mathrm{C}_{-}^{-\mathrm{CH}}, \mathrm{CH}-\mathrm{CH} . \mathrm{CH}_{2}, \mathrm{CH}_{8}$.

$I^{\prime \prime} . \mathrm{CHBr}_{2}, \mathrm{CBr}_{2}, \mathrm{CBr}_{2}, \mathrm{CHBr} \cdot\left(\mathrm{CH}_{2}\right)_{2}, \mathrm{CH}_{3}$.

$I^{\prime \prime \prime} \cdot \mathrm{CHBr}_{2} \cdot \mathrm{CBr}_{2}, \mathrm{CHBr}$. $\mathrm{CHBr} \cdot \mathrm{CHBr} \cdot \mathrm{CH}_{2} \cdot \mathrm{CH}_{8}$.

a. $\mathrm{CBr}_{2}=\mathrm{CBr}, \mathrm{CBr}_{2}=\mathrm{C}=\mathrm{CH} . \mathrm{CH}_{2} \cdot \mathrm{CH}_{3}=\mathrm{C}_{7} \mathrm{H}_{6} \mathrm{Br}_{4}$.

b. $\mathrm{CBr}_{2} \mathrm{C}-\mathrm{C}-\mathrm{C}=\mathrm{C}-\mathrm{CH} . \mathrm{CH}_{3}=\mathrm{C}_{7} \mathrm{H}_{4} \mathrm{Br}_{2}$.

b. $\mathrm{CBr}_{2}=\mathrm{CBr} . \mathrm{C} \equiv \mathrm{C} . \mathrm{CH}-\mathrm{CH} . \mathrm{CH}_{3}=\mathrm{C}_{7} \mathrm{H}_{5} \mathrm{Br}_{3}$.

b". $\mathrm{CBr}_{2-}=\mathrm{CBr} . \mathrm{C}=\mathrm{C} . \mathrm{CH}_{2}, \mathrm{CH}_{3}=\mathrm{C}_{7} \mathrm{H}_{5} \mathrm{Br}_{8}$. 
Erhalten wir $\mathrm{C}_{7} \mathrm{H}_{6} \mathrm{Br}_{4}$, so ist die Constitution dieses letzteren $a, \mathrm{C}_{7} \mathrm{H}_{9} \mathrm{Br} . \mathrm{Br}_{6}$ ist $\mathrm{I}^{\prime \prime}, \mathrm{C}_{7} \mathrm{H}_{9} \mathrm{Br}$ ist $1^{v}$ oder $2^{\prime \prime}$, folglich wird $\mathrm{C}_{7} \mathrm{H}_{12}$ den Combinationen 1 und 2 zukommen können. Da aber die Combination 1 zu sechs verschiedenen Combinationen von $\mathrm{C}_{7} \mathrm{H}_{9} \mathrm{Br}$ und $\mathrm{C}_{7} \mathrm{H}_{4}$, und die Combination 2 nur zu zwei Combinationen führen konnte, so ist es wahrscheinlicher, anzunehmen, dass $\mathrm{O}_{7} \mathrm{H}_{12}$ die Constitution 2 und nicht die Constitution 1 besitzt. $\mathrm{Zu}$ weiterer Reduction der für $\mathrm{C}_{7} \mathrm{H}_{12}$ noch als möglich übrig gebliebenen Combinationen 1 und 2 wird man in diesem ungünstigen Falle noch nach der zweiten Art der Reduction der Combinationen prüfen müssen. Wir werden daher die Constitution des $\left(\mathrm{C}_{7} \mathrm{H}_{14} \mathrm{Br}_{2}-1 \mathrm{BrH}\right)$ znerst bestimmen. Dieses kann durch successive Anlagerung von Brom und Abspaltung von Bromwasserstoff, sowie durch Oxydation geschehen. $\mathrm{C}_{7} \mathrm{H}_{12}$ kann noch $1, \mathrm{~d} . \mathrm{h}$.

$$
\mathrm{CH}_{2}-\mathrm{CH} . \mathrm{CH}_{\gamma}^{-} \mathrm{CH} .\left(\mathrm{CH}_{2}\right)_{2} \mathrm{CH}_{3}
$$

oder 2 sein, d. $\mathrm{h}$.

$$
\mathrm{CH}_{2}=\mathrm{C}_{\alpha}^{-} \mathrm{CH} .\left(\mathrm{CH}_{2}\right)_{3} \mathrm{CH}_{3} \text {. }
$$

Ergiebt sich nun, dass im $\left(\mathrm{C}_{1} \mathrm{H}_{14} \mathrm{Br}_{2}-1 \mathrm{BrH}\right)$ die Doppelbindung in $\beta$-Stellung sich befindet, so kann die Constitution des $\mathrm{C}_{7} \mathrm{H}_{12}$ nur 2 sein; ist die Doppelbindung in $\left(\mathrm{C}_{7} \mathrm{H}_{14} \mathrm{Br}_{2}-1 \mathrm{Br} \mathrm{H}\right)$ in $\gamma$-Stellung, so kann $\mathrm{C}_{7} \mathrm{H}_{12}$ nur 1 sein; dagegen kann $\mathrm{C}_{7} \mathrm{H}_{12}$ 1 und 2 sein, wenn die Doppelbindung in $\left(\mathrm{C}_{7} \mathrm{H}_{14} \mathrm{Br}_{2}-1 \mathrm{BrH}\right)$ in der $\alpha$-Stellung sich befindet. In diesem letzten Falle werden wir die Reduction nach der zweiten Art fortsetzen, und dazu die Constitution des $\left(\mathrm{C}_{7} \mathrm{H}_{12} \cdot \mathrm{Br}_{4}-1 \mathrm{BrH}\right),\left(\mathrm{C}_{7} \mathrm{H}_{12} \cdot \mathrm{Br}_{4}-2 \mathrm{BrH}\right)$ u. s. w. untersuchen, um indirect nach der zweiten Art die Combinationen 1 und 2 für $\mathrm{C}_{7} \mathrm{H}_{12}$ auf 1 oder 2 zu reduciren.

Gelangt man dagegen $\mathrm{zu} \mathrm{C}_{7} \mathrm{H}_{5} \mathrm{Br}_{3}$ oder zu $\mathrm{C}_{7} \mathrm{H}_{4} \mathrm{Br}_{2}$, so ist die Constitution des ersteren $b^{\prime}$ oder $b^{\prime \prime}$, des zweiten $b$; $\mathrm{C}_{7} \mathrm{H}_{9} \mathrm{Br} . \mathrm{Br}_{6}$ ist $\mathrm{I}^{\prime \prime \prime}, \mathrm{C}_{7} \mathrm{H}_{9} \mathrm{Br}$ ist $\mathrm{I}^{\mathrm{vI}}, \mathrm{C}_{7} \mathrm{H}_{12} \cdot \mathrm{Br}_{4}$ ist $\mathrm{I}$, und die gesuchte Combination für $\mathrm{C}_{7} \mathrm{H}_{12}$ ist $1, \mathrm{~d}$. h.

$$
\mathrm{CH}_{2}-\mathrm{CH} \text {. } \mathrm{CH}^{-} \mathrm{CH} .\left(\mathrm{CH}_{2}\right)_{2} \mathrm{CH}_{3} \text {. }
$$

B) Angenommen sei, dass bei der Anlagerung von Brom an $\mathrm{C}_{7} \mathrm{H}_{12}$ und Abspaltung von Bromwasserstof wir nicht nur $\mathrm{C}_{7} \mathrm{H}_{9} \mathrm{Br}$, sondern $\mathrm{C}_{7} \mathrm{H}_{8}$ gewinnen konnten. $\mathrm{C}_{7} \mathrm{H}_{8}$ kann, wie oben aus der ersten Tabelle ersichtlich ist, 
nur noch eine der theoretischen Constitutionsformeln $1^{\prime}, 1^{\prime \prime}, 1^{\prime \prime \prime}$ und $3^{\prime}$ besitzen. Aus diesen Combinationen sind $1^{\text {IV }}$ und $3^{\prime}$ identisch. $\mathrm{C}_{7} \mathrm{H}_{12}$ kann daher nur noch 1 oder 3 sein. $\mathrm{Zu}$ weiterer Reduction der Combinationen 1 und 3 wird es genügen, das Verhalten des $\mathrm{C}_{7} \mathrm{H}_{8}$ gegen ammoniakalisches Kupferchlorür zu untersuchen, denn $1^{1 \mathrm{~V}}=3^{\prime}$ fällt kein ammoniakalisches $\mathrm{Cu}_{2} \mathrm{Cl}_{2}, 1^{\prime}$ und $1^{\prime \prime}$ dagegen fällen. Im ersten Falle werden wir noch nach der zweiten Art Reduction der Combinationen greifen, im zweiten Falle aber ist $\mathrm{C}_{7} \boldsymbol{H}_{8} 1^{\prime}$ oder $1^{\prime \prime}$ (welche durch eine einmalige Anlagerung von Brom und Abspaltung von Bromwasserstoff von einander unterschieden werden müssen), $\mathrm{C}_{7} \mathrm{H}_{12} \cdot \mathrm{Br}_{4}$ ist jedenfalls $\mathrm{I}$, und die gesuchte Constitution des $\mathrm{C}_{7} \mathrm{H}_{12}$ ist 1, d. h. $\mathrm{CH}_{2}-\mathrm{CH}$. CH $\mathrm{CH} .\left(\mathrm{CH}_{2}\right)_{2} \mathrm{CH}_{3}$.

Auf Grund der einmaligen oder zweimaligen Anlagerung von Brom und Abspaltung von Bromwasserstoff haben wir in den überaus meisten Fällen die Constitution des $\mathrm{C}_{7} \mathrm{H}_{12}$ bestimmt, ohne uns zu der $\mathrm{z}$ weiten Art der Reduction $\mathrm{zu}$ wenden. Hierbei ermittelten wir gleichzeitig die Constitution von $\mathrm{C}_{7} \mathrm{H}_{12} . \mathrm{Br}_{4}$ und $\mathrm{C}_{7} \mathrm{H}_{9} \mathrm{Br}, \mathrm{C}_{7} \mathrm{H}_{9} \mathrm{Br} . \mathrm{Br}_{6}$ oder $\mathrm{C}_{7} \mathrm{H}_{8}$, $\mathrm{C}_{7} \mathrm{H}_{8} \cdot \mathrm{Br}_{8}$ - das sind Körper mit höherem Halogengehalt, mit mehreren doppelten und dreifachen Bindungen.

\section{Beispiel 2.}

Es soll die Constitution von $\left(\mathrm{C}_{6} \mathrm{H}_{11} \mathrm{Br}_{3}-3 \mathrm{BrH}\right)=\mathrm{C}_{6} \mathrm{H}_{8}$ bestimmt werden, wenn dieses letztere durch Abspaltung von drei Molekülen Bromwasserstoff aus $\left(\mathrm{CH}_{3}\right)_{2} \mathrm{CBr}$. $\mathrm{CHBr}$. CHBr. $\mathrm{CH}_{3}$ entstanden ist.

Für $\mathrm{C}_{6} \mathrm{H}_{8}$ sind drei Combinationen möglich:

1.

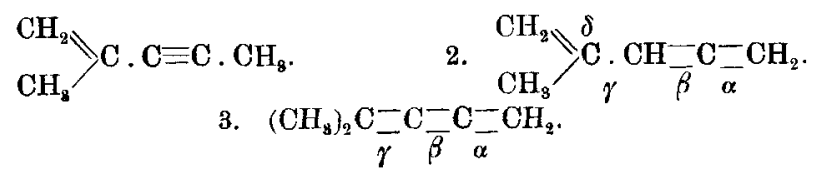

I.<smiles>[10BH2][13CH](C)[13CH2][13CH2][13CH2]C</smiles>

II.

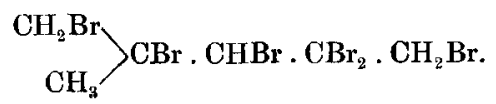

III. $\left(\mathrm{CH}_{8}\right)_{2} \mathrm{CBr} \cdot \mathrm{CBr}_{2}, \mathrm{CBr}_{2}, \mathrm{CH}_{2} \mathrm{Br}$.

Journal f. prakt. Chemie [a] Bd. 44. 
Wildermann: Ueber eine Methode etc.

1. $\mathrm{CHBr}$

$\mathrm{C} \cdot \mathrm{CBr}_{2} \cdot \mathrm{C} \equiv \mathrm{CH}$

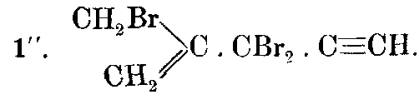

2. $\underset{\mathrm{CH}_{3}}{\mathrm{CHBr}} \searrow \mathrm{C} \cdot \mathrm{CHBr} . \mathrm{C} \equiv \mathrm{CBr}$.

2". $\stackrel{\mathrm{CH}_{2} \mathrm{Br}}{\mathrm{CH}_{2}} \geqslant \mathrm{C} . \mathrm{CHBr} . \mathrm{C} \equiv \mathrm{CBr}$.

$2^{\prime \prime \prime} . \quad \underset{\mathrm{CH}_{3}}{\mathrm{CH}_{2} \mathrm{Br}}>\underset{\gamma}{\mathrm{C}}-\underset{\beta}{\mathrm{CBr}}+\underset{\alpha}{\mathrm{C}} \equiv \mathrm{CBr}$

$2^{\text {IV }}$.

$\stackrel{\mathrm{CH}_{2} \mathrm{Br}}{\mathrm{CH}_{2}} \geqslant \mathrm{C} . \mathrm{CBr}_{-}^{-} \mathrm{C}_{-}^{-} \mathrm{CHBr}$.

2v. $\underset{\mathrm{CH}_{3}}{\mathrm{CHBr}} \ \mathrm{C} \cdot \mathrm{CBr}_{-} \mathrm{C}_{-} \mathrm{CHBr} . \quad 3 "$.<smiles></smiles>

Gelangt man zu $\mathrm{C}_{6} \mathrm{H}_{5} \mathrm{Br}_{3}$, welches ammoniakalisches Kupferchlorür fällt, so ist die Constitution desselben I' oder 1". Die Constitution des $\mathrm{C}_{6} \mathrm{H}_{8} \mathrm{Br}_{6}$ wird I sein, die gesuchte Constitution des $\mathrm{C}_{6} \mathrm{H}_{8}$ wird daher 1 , d. h.

$$
\mathrm{CH}_{2} \searrow \mathrm{C} . \mathrm{C}=\mathrm{C} \cdot \mathrm{CH}_{3} \text { sein. }
$$

Wird $\mathrm{C}_{6} \mathrm{H}_{5} \mathrm{Br}_{3}$ durch ammoniakalisches Kupferchlorür nicht gefällt, so sind die Combinationen $1^{\prime}$ und $1^{\prime \prime}$ für $\mathrm{C}_{6} \mathrm{H}_{5} \mathrm{Br}_{3}$ ausgeschlossen und es sind noch die Combinationen $2^{\prime}, 2^{\prime \prime}, 2^{\prime \prime \prime}, 2^{\mathrm{Iv}}, 2^{\mathrm{v}}$ und $3^{\prime}$ möglich. Addirt man Brom zu diesen letzteren bis zur Sättigung und bewirkt man das Maximum der Bromwasserstoffabspaltungen, so werden, wie das aus der obigen Tabelle leicht ersichtlich ist, $2^{\prime}, 2^{\prime \prime}, 2^{1 \mathrm{v}}, 2^{\mathrm{v}}$ zwei Bromwasserstoffe, $2^{\prime \prime \prime}$ und $3^{\prime}$ dagegen nur ein Bromwasserstoffatom abspalten können. Im ersteren Falle werden wir zu $\mathrm{C}_{6} \mathrm{H}_{3} \mathrm{Br}_{7}$ gelangen, im zweiten zu $\mathrm{C}_{6} \mathrm{H}_{4} \mathrm{Br}_{8}$.

Gelangt man zu $\mathrm{C}_{6} \mathrm{H}_{3} \mathrm{Br}_{7}$, so kann $\mathrm{C}_{6} \mathrm{H}_{5} \mathrm{Br}_{3} 2^{\prime}, 2^{\prime \prime}, 2^{\mathrm{Iv}}, 2^{\mathrm{v}}$ sein; $\mathrm{C}_{8} \mathrm{H}_{8} \mathrm{Br}_{6}$ wird jedenfalls II sein, und die gesuchte Constitution des $\mathrm{C}_{6} \mathrm{H}_{8}$ wird daher 2 sein, d. h.

$$
\mathrm{CH}_{3} \gg \mathrm{C} . \mathrm{CH}_{2} \mathrm{CH}_{2}-\mathrm{CH}_{2} \ldots
$$

Gelangt man dagegen zu $\mathrm{C}_{8} \mathrm{H}_{4} \mathrm{Br}_{8}$, so ist die Formel des $\mathrm{C}_{6} \mathrm{H}_{5} \mathrm{Br}_{3} 2^{\prime \prime \prime}$ oder $3^{\prime} ; \mathrm{C}_{6} \mathrm{H}_{8}$ kann noch immer 2 oder 3 sein, 
Wildermann: Ueber eine Methode etc.

und wir werden noch die zweite Art Reduction anwenden müssen. Zunächst müssen wir aber annehmen, dass $\mathrm{C}_{6} \mathrm{H}_{5} \mathrm{Br}_{3}$ wahrscheinlich die Constitution $3^{\prime}$ besitzt, da $\mathrm{C}_{6} \mathrm{H}_{8} . \mathrm{Br}_{6}$ III nur zu $\mathrm{C}_{6} \mathrm{H}_{5} \mathrm{Br}_{3} 3^{\prime}$, dagegen $\mathrm{C}_{6} \mathrm{H}_{8} \cdot \mathrm{Br}_{6}$ II ausser zu 2"' noch zu vier anderen Constitutionsformeln führen konnte.

In den meisten Fällen genügt also eine einmalige oder $\mathrm{zweimalige}$ Anlagerung von Brom und Abspaltung von Bromwasserstoff, um die Constitution des $\mathrm{C}_{6} \mathrm{H}_{8}$, ohne Anwendung der zweiten Art Reduction, zu erkennen.

\section{Beispiel 3 und 4.}

Bei der Anlagerung ron Brom an das aus Methyldiäthylearbinoljodür bei der Behandlung mit alkoholischem Kali erhaltene Dimethyläthyläthylen

$$
\underset{\mathrm{CH}_{3}}{\mathrm{CH}_{3} . \mathrm{CH}_{2}}>\mathrm{C}_{-\mathrm{CH} . \mathrm{CH}_{3}}
$$

entsteht $\mathrm{C}_{6} \mathrm{H}_{12} \mathrm{Br}_{2}$ von der Constitution

$$
\mathrm{CH}_{3} \cdot \mathrm{CH}_{2}>\mathrm{CBr} . \mathrm{CHBr} . \mathrm{CH}_{3} \text {. }
$$

Es soll die Constitutionsformel des $\left(\mathrm{C}_{6} \mathrm{H}_{12} \mathrm{Br}_{2}-2 \mathrm{BrH}\right)$, sowie des $\left(\mathrm{C}_{6} \mathrm{H}_{1,} \mathrm{Br}_{2}-1 \mathrm{BrH}\right)$ ermittelt werden.

A) $\left(\mathrm{C}_{6} \mathrm{H}_{12} \mathrm{Br}_{2}-2 \mathrm{BrH}\right)=\mathrm{C}_{6} \mathrm{H}_{10}$ kann eine der folgenden Formeln besitzen:

1.

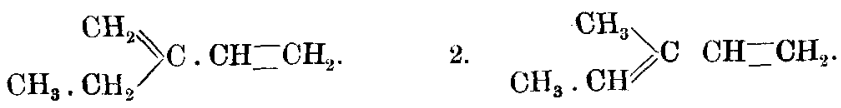

3

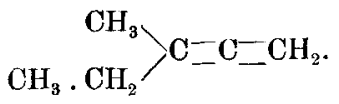

1 .

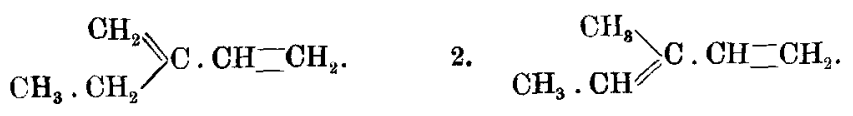

3.

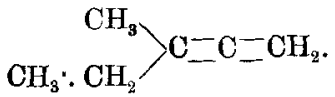


484 Wildermann: Ueber eine Methode etc.

$$
\begin{aligned}
& \text { I. } \underset{\mathrm{CH}_{3} \cdot \mathrm{CH}_{2}}{\mathrm{CH}_{2} \mathrm{Br}}>\mathrm{CBr} \cdot \mathrm{CHBr} \text {. } \mathrm{CH}_{2} \mathrm{Br}
\end{aligned}
$$

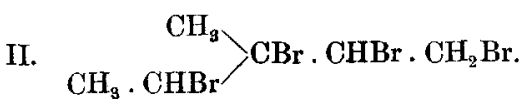

$$
\begin{aligned}
& \text { III. } \underset{\mathrm{CH}_{3} \cdot \mathrm{CH}_{2}}{\mathrm{CH}_{\mathrm{B}}}>\mathrm{CBr} \cdot \mathrm{CBr}_{2} \cdot \mathrm{CH}_{2} \mathrm{Br} \text {. }
\end{aligned}
$$

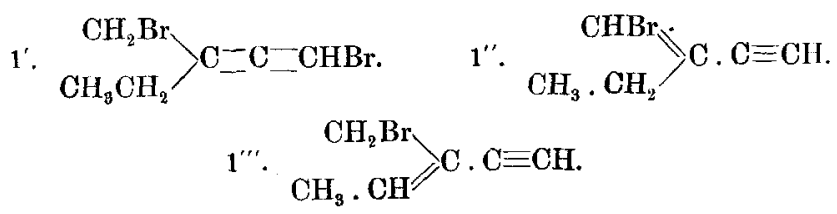

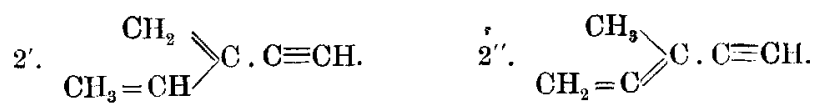

$$
\begin{aligned}
& \text { 2"'. } \underset{\mathrm{CH}_{2}=\mathrm{CH}}{\mathrm{CH}_{3}}>\mathrm{C}_{-}^{-} \mathrm{C}_{-}^{-} \mathrm{CHBr} \text {. }
\end{aligned}
$$

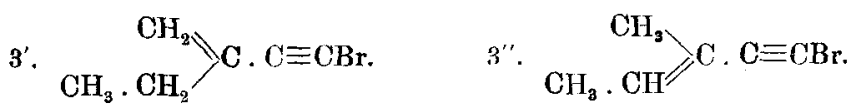

Gelangt man zu $\mathrm{O}_{6} \mathrm{~B}_{8} \mathrm{Br}_{2}$, so ist die Constitution dieses letzteren $1^{\prime}$, gelangt man zu $\mathrm{C}_{8} \mathrm{H}_{7} \mathrm{Br}$, welches ammoniakalisches Kupferchlorür fällt, so ist die Constitution dieses letzteren $1^{\prime \prime}$ oder $1^{\prime \prime \prime}$. In beiden Fällen ist die Constitution des $\mathrm{C}_{6} \mathrm{H}_{10} \mathrm{Br}_{4} \mathrm{I}$, und die gesuchte Constitution des $\mathrm{C}_{6} \mathrm{H}_{10}$ wird 1 , d. h. ${ }_{\mathrm{CH}_{3} . \mathrm{OH}_{2}}^{\mathrm{CH}_{2}}>\mathrm{C} . \mathrm{CH}^{-} \mathrm{CH}_{2}$ sein.

Gelangt man dagegen zu $\mathrm{C}_{6} \mathrm{H}_{6}$, (welches ammoniakalisches Kupferchlorür fällt), so ist die Constitution dieses letzteren $2^{\prime}$ oder 2", die Constitution von $\mathrm{C}_{6} \mathrm{H}_{10} \mathrm{Br}_{4}$ ist $\mathrm{II}$, die 'gesuchte Constitution des $\mathrm{C}_{6} \mathrm{H}_{10}$ ist 2, d. h. $\mathrm{CH}_{3} . \mathrm{CH}_{3} \geqslant \mathrm{C} . \mathrm{CH}_{-}^{-} \mathrm{CH}_{2}$. Gelangt man zu $\mathrm{C}_{6} \mathrm{H}_{7} \mathrm{Br}$, welches ammoniakalisches Kupferchlorïr nicht fällt, so ist die Constitution dieses letzteren 3 ', $3^{\prime \prime}$ oder $2^{\prime \prime \prime}$. 
Wildermann: Ueber eine Methode etc.

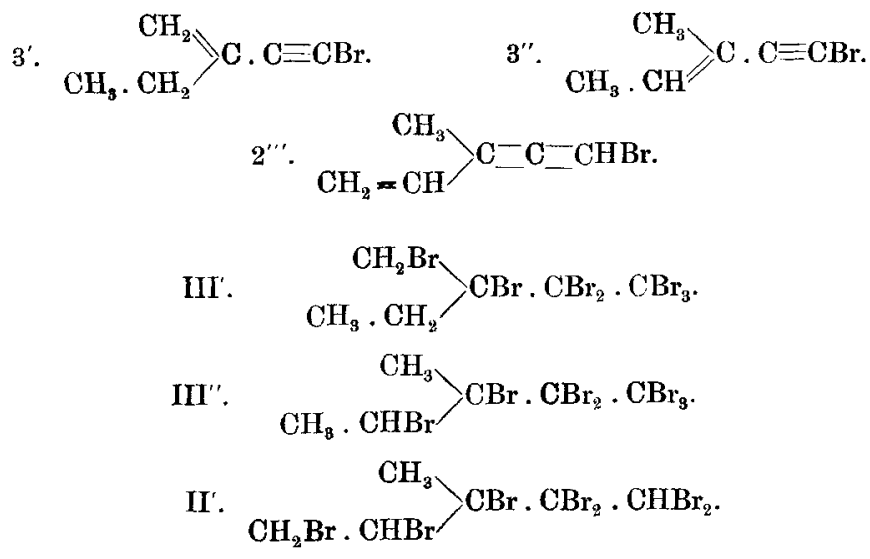

$3^{\prime \prime \prime}$. Spaltet $1 \mathrm{BrH}$ ab. $\quad 3^{\mathrm{IV}}$. Spaltet $2 \mathrm{BrH}$ ab. $2^{\text {IV }}$. Spaltet 3 oder $4 \mathrm{BrH}$ ab.

Gelangt man $\mathrm{zu} \mathrm{C}_{6} \mathrm{H}_{6} \mathrm{Br}_{6}$, so ist dieses letztere $3^{\prime \prime}$, $\mathrm{C}_{6} \mathrm{H}_{7} \mathrm{Br}_{7}$ ist $\mathrm{III}^{\prime}, \mathrm{C}_{6} \mathrm{H}_{7} \mathrm{Br}$ ist $\mathbf{3}^{\prime}, \mathrm{C}_{6} \mathrm{H}_{10} \mathrm{Br}_{4}$ ist III, und die gesuchte Constitution des $\mathrm{C}_{6} \mathrm{H}_{10}$ ist 3 , d. h.

$$
\mathrm{CH}_{3} . \mathrm{CH}_{2}>\mathrm{CH}_{3}^{-} \mathrm{CH}_{2} \text {. }
$$

Gelangt man zu $\mathrm{C}_{6} \mathrm{H}_{5} \mathrm{Br}_{5}$, so ist dieses letztere $3^{1 \mathrm{v}}, \mathrm{C}_{6} \mathrm{H}_{7} \mathrm{Br}_{7}$ ist $\mathrm{CH}_{3} \cdot \mathrm{CHBr}_{3}>\mathrm{CBr} . \mathrm{CBr}_{2} \cdot \mathrm{CBr}_{3}, \mathrm{C}_{6} \mathrm{H}_{7} \mathrm{Br}$ ist $3^{\prime \prime}, \mathrm{C}_{6} \mathrm{H}_{10} \mathrm{Br}_{4}$. ist III, und $\mathrm{C}_{6} \mathrm{H}_{10}$ ist wieder $\mathrm{CH}_{3} \cdot \mathrm{CH}_{2}>\mathrm{C}-\mathrm{C}_{-}-\mathrm{CH}_{2}$. Erhält man dagegen $\mathrm{C}_{6} \mathrm{H}_{4} \mathrm{Br}_{4}$, oder $\mathrm{C}_{6} \mathrm{H}_{3} \mathrm{Br}_{3}$, so ist das erstere $\mathrm{CHBr}=\mathrm{C}^{\mathrm{CH}_{3}}>\mathrm{C} \cdot \mathrm{CBr}_{-} \mathrm{CBr}_{2}$, das zweite $\begin{array}{r}\mathrm{CH}_{2} \\ \mathrm{CH}=\mathrm{C}\end{array} \mathrm{CBr}_{-\mathrm{CBr}_{2}}$; $\mathrm{C}_{6} \mathrm{H}_{7} \mathrm{Br}_{7}$ ist jedenfalls II', $\mathrm{C}_{6} \mathrm{H}_{7} \mathrm{Br}$ ist $2^{\prime \prime \prime}, \mathrm{C}_{6} \mathrm{H}_{10} \mathrm{Br}_{4}$ ist II, und die gesuchte Constitution des $\mathrm{C}_{6} \mathrm{H}_{10}$ ist 2 , d. h.

$$
\begin{array}{r}
\mathrm{CH}_{3} \\
\mathrm{CH}_{3} \cdot \mathrm{CH}^{\prime}
\end{array}
$$

Allen Umständen nach muss somit die Constitution des $\mathrm{C}_{6} \mathrm{H}_{10}$ auf Grund der einmaligen oder zweimaligen Anlagerung von Brom und Abspaltung von Bromwasserstoff bestimmt werden können. 
B) Wir bestimmen jetzt die Constitution von

$$
\left(\mathrm{C}_{6} \mathrm{H}_{12} \mathrm{Br}_{2}-1 \mathrm{BrH}\right)=\mathrm{C}_{6} \mathrm{H}_{11} \mathrm{Br} \text {. }
$$

1. Ist die Constitution des $\mathrm{C}_{6} \mathrm{H}_{10} 1, \mathrm{~d}$. h.

$$
\mathrm{CH}_{3} . \mathrm{CH}_{2} \searrow \mathrm{CH} \cdot \mathrm{CH}_{-}^{-} \mathrm{CH}_{2} \text {, }
$$

so kann $\mathrm{C}_{6} \mathrm{H}_{11} \mathrm{Br}$ 1. ${ }_{\mathrm{CH}_{3} \cdot \mathrm{CH}_{2}}^{\mathrm{CH}_{2}}>\mathrm{C} \cdot \mathrm{CHBr} \mathrm{CH}_{3}$ oder

$$
\text { 2. } \underset{\mathrm{CH}_{3} \cdot \mathrm{CH}_{2}}{\mathrm{CH}_{3}}>\mathrm{CBr} \cdot \mathrm{CH}_{-}^{-} \mathrm{CH}_{2} \text { sein. }
$$

$$
\begin{aligned}
& \text { 1. } \underset{\mathrm{CH}_{3}, \mathrm{CH}_{2}}{\mathrm{CH}_{2}} \backslash \mathrm{C} \cdot \mathrm{CHBr} \cdot \mathrm{CH}_{3} . \quad \text { 2. }{ }_{\mathrm{CH}_{3}, \mathrm{CH}_{2}}^{\mathrm{CH}_{3}}>\mathrm{CBr} \cdot \mathrm{CH}^{-} \mathrm{CH}_{2} \text {. }
\end{aligned}
$$

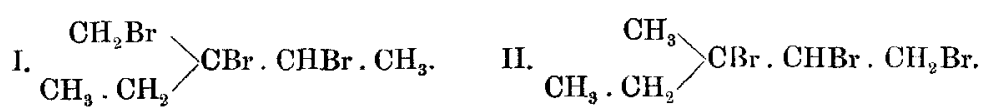

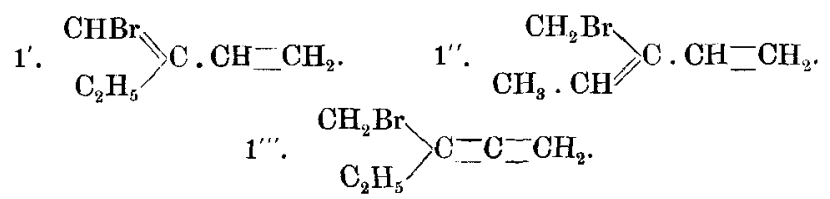

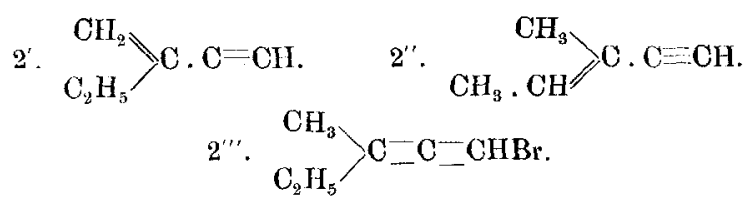

Gelangt man zu $\mathrm{C}_{6} \mathrm{H}_{8}$ (welches ammoniakalisches $\mathrm{Cu}_{2} \mathrm{Cl}_{2}$ fällen wird) so ist die Constitution dieses letzteren $2^{\prime}$ oder $2^{\prime \prime}$, $\mathrm{C}_{6} \mathrm{H}_{11} \mathrm{Br}_{3}$ ist II, und die gesuchte Constitution des $\mathrm{C}_{6} \mathrm{H}_{11} \mathrm{Br}$

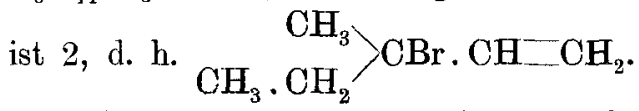

Will man noch nebenbei untersuchen, welche Constitution das $\mathrm{C}_{6} \mathrm{H}_{8}$ besitzt, so genügt es wieder, Brom zu addiren und Bromwasserstoff abzuspalten: $2^{\prime}$ wird nur $2 \mathrm{BrH}$ abspalten können, $2^{\prime \prime}$ wird dagegen $3 \mathrm{BrH}$ abspalten. Gewinnt man $\mathrm{C}_{6} \mathrm{H}_{9} \mathrm{Br}$, so kann dieses letztere $1^{\prime}, 1^{\prime \prime \prime}$ und $2^{\prime \prime}$ sein. 
Wildermann: Ueber eine Methode ete.

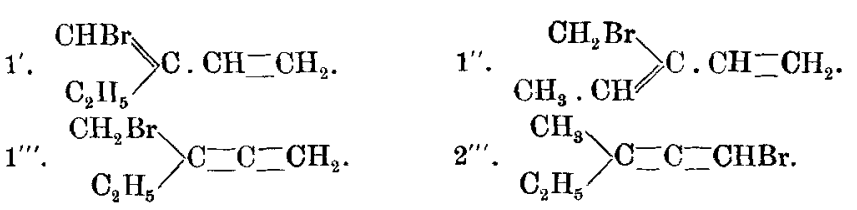<smiles>C#CC(Br)(CCBr)C(Br)[C@H](Br)CCBr</smiles><smiles>C#CC(Br)C=CCCC(=C)C=CC(=C=C)C(Br)C=C</smiles>

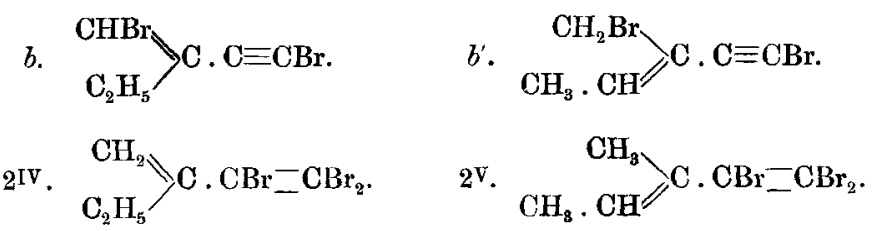

Wie aus der Tabelle ersichtlich ist, muss $\mathrm{C}_{6} \mathrm{H}_{11} \mathrm{Br}$ die Constitution 1 besitzen, wenn $\mathrm{C}_{6} \mathrm{H}_{9} \mathrm{Br} \mathrm{Br}_{4}$ nur mehr als $2 \mathrm{BrH}$ abspaltet.

Gelangt man $\mathrm{zu} \mathrm{C}_{6} \mathrm{H}_{5} \mathrm{Br}$, so ist die Constitution dieses letzteren $a^{\prime}$ oder $a^{\prime \prime}, \mathrm{C}_{6} \mathrm{H}_{9} \mathrm{Br}_{5}$ ist $\mathrm{I}^{\prime \prime}, \mathrm{C}_{6} \mathrm{H}_{9} \mathrm{Br}$ ist $1^{\prime \prime}, \mathrm{C}_{6} \mathrm{H}_{11} \mathrm{Br}_{3}$ ist $I$, und die gesuchte Constitution des $\mathrm{C}_{6} \mathrm{H}_{11} \mathrm{Br}$ ist $1, \mathrm{~d}$. $\mathrm{h}$. $\mathrm{CH}_{2}>\mathrm{C} . \mathrm{CHBr}_{2} \mathrm{CH}_{3}$. Erhält man $\mathrm{O}_{6} \mathrm{H}_{6} \mathrm{Br}_{2}$, welches ammoniakalisches Küpferchlorür fällt, so ist dieses letztere $1^{\mathbf{v}}$ oder $1^{\mathrm{v} 1}, \mathrm{C}_{6} \mathrm{H}_{9} \mathrm{Br}_{5}$ ist $\mathrm{I}^{\prime}, \mathrm{C}_{6} \mathrm{H}_{9} \mathrm{Br}$ ist $1^{\prime}, \mathrm{C}_{6} \mathrm{H}_{11} \mathrm{Br}_{3}$ ist $\mathrm{I}$, und $\mathrm{C}_{6} \mathrm{H}_{11} \mathrm{Br}$ wieder 1. Erhält man $\mathrm{C}_{6} \mathrm{H}_{6} \mathrm{Br}_{2}$, welches ammoniakalisches Kupferchlorür nicht fällt, so kann dieses letztere $a, b, b^{\prime}$ sein. Ergiebt sich bei weiterer Anlagerung von Brom und Maximal- 
abspaltung von Bromwasserstoff, dass $\left(\mathrm{C}_{6} \mathrm{H}_{6} \mathrm{Br}_{2}+6 \mathrm{Br}\right) 3$ oder $4 \mathrm{BrH}$ abspaltet, so ist $\mathrm{O}_{6} \mathrm{H}_{6} \mathrm{Br}_{2}$ von der Constitution $a$, spaltet es dagegen nur 1 oder 2 Bromwasserstoffatome ab, so kann es $b$ (spaltet $1 \mathrm{BrH} \mathrm{ab}$ ) oder $b^{\prime}$ (spaltet $2 \mathrm{BrH}$ ab) sein. Ist $\mathrm{C}_{6} \mathrm{H}_{6} \mathrm{Br}_{2}$ $a$, so ist $\mathrm{C}_{6} \mathrm{H}_{9} \mathrm{Br}_{5} \mathrm{I}^{\prime \prime}, \mathrm{C}_{6} \mathrm{H}_{9} \mathrm{Br}$ ist $1^{\prime \prime}, \mathrm{C}_{6} \mathrm{H}_{11} \mathrm{Br}_{3}$ ist $\mathrm{I}$, und $\mathrm{C}_{6} \mathrm{H}_{11} \mathrm{Br}$ ist wieder 1 . Ist $\mathrm{C}_{6} \mathrm{H}_{6} \mathrm{Br}_{2} b$ oder $b^{\prime}$, so wird $\mathrm{C}_{6} \mathrm{H}_{9} \mathrm{Br}_{5} \mathrm{I}^{\prime \prime \prime}$ sein, $\mathrm{C}_{6} \mathrm{H}_{9} \mathrm{Br}$ wird $1^{\prime \prime \prime}, \mathrm{C}_{6} \mathrm{H}_{11} \mathrm{Br}_{3}$ wird $\mathrm{I}$, und $\mathrm{C}_{6} \mathrm{H}_{11} \mathrm{Br}$ wieder 1 .

Spaltet $\mathrm{C}_{6} \mathrm{H}_{9} \mathrm{Br}_{5}$ nur $2 \mathrm{BrH}$ ab, so kann dieses letztere $1^{1 \mathrm{v}}, 2^{\mathrm{Nv}}, 2^{\mathrm{v}}$ sein.

$$
\begin{aligned}
& \text { 1'v. } \begin{array}{r}
\mathrm{CHBr}_{2} \\
\mathrm{CH}_{3}, \mathrm{CH}_{2}
\end{array}>\mathrm{C}^{-\mathrm{C}}-\mathrm{CHBr} . \quad 2^{\mathrm{IV}} \cdot \begin{array}{c}
\mathrm{CH}_{2} \\
\mathrm{C}_{2} \mathrm{H}_{6}
\end{array}>\mathrm{C} \cdot \mathrm{CBr}_{-}-\mathrm{CBr}_{2} . \\
& 2 \text { v. } \underset{\mathrm{CH}_{3} \cdot \mathrm{CH}^{2}}{\mathrm{CH}_{3}}>\mathrm{C} \cdot \mathrm{CBr}_{-}^{-} \mathrm{CBr}_{2} \text {. }
\end{aligned}
$$

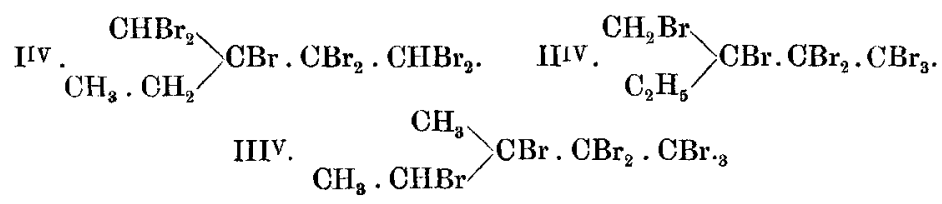

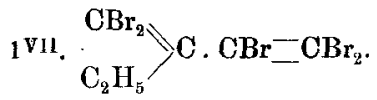

$$
\begin{aligned}
& \text { 1 }{ }^{\mathrm{VIII}} \underset{\mathrm{CH}_{3} \cdot \mathrm{CH}_{2}}{\mathrm{CHBr}_{2}}>\mathrm{C} . \mathrm{CBr}_{-}^{-} \mathrm{CBr}_{2} \text {. } \\
& 2^{\mathrm{VI}} \cdot \underset{\mathrm{C}_{2} \mathrm{H}_{5}}{\mathrm{CHBr}} \backslash \mathrm{C} \cdot \mathrm{CBr}_{2} \cdot \mathrm{CBr}_{3} \text {. } \\
& 2^{\text {VII. }} \underset{\mathrm{CH}_{3} . \mathrm{CH}}{\mathrm{CH}_{2} \mathrm{Br}}>\mathrm{C} \cdot \mathrm{CBr}_{2} \cdot \mathrm{CBr}_{3} \text {. }
\end{aligned}
$$

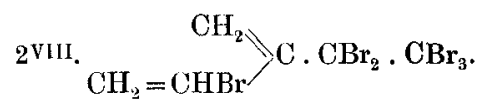

$$
\begin{aligned}
& { }^{2 \mathrm{IX}} . \underset{\mathrm{CH}_{2}=\mathrm{C}}{\mathrm{CH}_{3}}>\mathrm{C} \cdot \mathrm{CBr}_{2} \cdot \mathrm{CBr}_{\mathrm{s}} \text {. }
\end{aligned}
$$

Gewinnt man $\mathrm{C}_{6} \mathrm{H}_{6} \mathrm{Br}_{6}$, d. h. spaltet $\mathrm{C}_{6} \mathrm{H}_{7} \mathrm{Br}_{7}$ nur $1 \mathrm{BrH}$ $a b$, so ist $\mathrm{C}_{6} \mathrm{H}_{6} \mathrm{Br}_{6} 2^{\mathrm{vl}}$ oder $2^{\mathrm{vII}}$, welche aus $\mathrm{C}_{6} \mathrm{H}_{7} \mathrm{Br}_{7} \mathrm{II}^{\mathrm{vV}}$ entstanden, $\mathrm{C}_{6} \mathrm{H}_{7} \mathrm{Br}_{3}$ wird $2^{\mathrm{IV}}$ sein, $\mathrm{C}_{6} \mathrm{H}_{9} \mathrm{Br}_{5}$ ist $\mathrm{II}^{\prime \prime \prime}, \mathrm{C}_{6} \mathrm{H}_{8} \mathrm{Br}$ ist $2^{\prime \prime \prime}, \mathrm{C}_{8} \mathrm{H}_{11} \mathrm{Br}_{3}$ ist IT, und die gesuchte Constitution für $\mathrm{C}_{6} \mathrm{H}_{11} \mathrm{Br}$ ist 2, d. h. $\underset{\mathrm{C}_{2} \mathrm{H}_{5}}{\mathrm{CH}_{3}}>\mathrm{CBr} . \mathrm{CH} \quad \mathrm{CH}_{2}$. Gelangt man dagegen zu $\mathrm{C}_{6} \mathrm{H}_{5} \mathrm{Br}_{5}$, d. h. spaltet $\mathrm{C}_{6} \mathrm{H}_{7} \mathrm{Br}_{7} 2 \mathrm{BrH}$ ab, so kann das $1^{\mathrm{VII}}, 1^{\mathrm{VIII}}$ und $2^{\mathrm{VIII}}, 2^{\text {IX }}$ sein. Bei weiterer Addition von Brom und Einwirkung von alkoholischem Kali kann $1^{\mathrm{vII}}$ nur $1 \mathrm{BrH}$, und $1^{\mathrm{v} I I}$ nur $2 \mathrm{BrH}$ abspalten, dagegen werden $2^{\text {VIII }}$ und $2^{\text {IX }}$ jedes $3 \mathrm{BrH}$ abspalten können. Spaltet daher 
Wildermann: Ueber eine Methode etc.

$\left(\mathrm{C}_{6} \mathrm{H}_{5} \mathrm{Br}_{5}+4 \mathrm{Br}\right)$ ein oder zwei $\mathrm{BrH}$ ab, so ist $\mathrm{C}_{6} \mathrm{H}_{5} \mathrm{Br}_{5} 1^{\mathrm{VII}}$ oder $1^{\mathrm{VIII}}, \mathrm{C}_{\mathrm{n}} \mathrm{H}_{7} \mathrm{Br}_{7}$ ist $\mathrm{I}^{\mathrm{IV}}, \mathrm{C}_{8} \mathrm{H}_{7} \mathrm{Br}_{3}$ ist 1v $^{\mathrm{Iv}}, \mathrm{C}_{6} \mathrm{H}_{9} \mathrm{Br}$ ist $1^{\prime}, \mathrm{C}_{8} \mathrm{H}_{11} \mathrm{Br}_{3}$ ist $\mathrm{I}$, und die gesuchte Constitution des $\mathrm{C}_{6} \mathrm{H}_{11} \mathrm{Br}$ ist $1, \mathrm{~d}$. h. $\mathrm{CH}$ $\mathrm{C}_{2} \mathrm{H}_{5}$ C. $\mathrm{CHBr} . \mathrm{CH}_{3}$. Spaltet $\left(\mathrm{C}_{6} \mathrm{H}_{5} \mathrm{Br}_{5}+4 \mathrm{Br}\right)$ drei $\mathrm{BrH}$, so ist $\mathrm{C}_{6} \mathrm{H}_{5} \mathrm{Br}_{5} 2^{\mathrm{v} 11 \mathrm{I}}$ oder $2^{\mathrm{IX}}$ and durch entsprechendes Durchgehen der Zwischenglieder wird sich ergeben, dass die gesuchte Constitution des $\mathrm{C}_{6} \mathrm{H}_{11} \mathrm{Br}$ 2, d.h. $\mathrm{CH}_{3} \cdot \mathrm{CH}_{2} \backslash \mathrm{CBr}$. $\mathrm{OH}=\mathrm{CH}_{2}$ ist.

Wie man sieht, muss man theoretisch unter allen Umständen die Constitution des $\left.\mathrm{C}_{8} \mathrm{H}_{11} \mathrm{Br}^{1}\right)$ bestimmen können, wenn $\mathrm{C}_{6} \mathrm{H}_{10}$ von der Constitution $\mathrm{1}_{\text {s d. h. }} \underset{\mathrm{C}_{2} \mathrm{H}_{5}}{\mathrm{CH}_{2}}>\mathrm{O}$. $\mathrm{CH}_{-} \mathrm{CH}_{2}$ ist.

2. Ist die Constitution des $\mathrm{C}_{6} \mathrm{H}_{20} 2, \mathrm{~d}$. h. $\mathrm{CH}_{3}$. $\mathrm{CH}_{3} \backslash \mathrm{C} . \mathrm{CH}-\mathrm{CH}_{2}$, so kann $\mathrm{C}_{6} \mathrm{H}_{11} \mathrm{Br}$ 1. ${ }_{\mathrm{CH}_{3} . \mathrm{CH}^{2}}^{\mathrm{CH}_{3}}>\mathrm{C} . \mathrm{CHBr} . \mathrm{CH}_{3}$ oder ${ }_{2 .}{ }_{\mathrm{C}_{2} \mathrm{H}_{5}}^{\mathrm{CH}_{3}}>\mathrm{CBr} . \mathrm{CH}^{-} \mathrm{CH}_{2}$ sein.<smiles>CC=C(CC)C([13CH3])[14CH3]</smiles>

I. ${ }_{\mathrm{CH}_{3} .}{ }_{\mathrm{CHBr}} \mathrm{CH}_{3}>\mathrm{CBr}$. $\mathrm{CHBr}$. $\mathrm{CH}_{8}$.

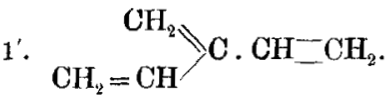

2. $\stackrel{\mathrm{CH}_{3}}{\mathrm{C}_{2} \mathrm{H}_{5}}>\mathrm{CBr} \cdot \mathrm{CH}_{-}^{-} \mathrm{CH}_{2}$.

II. $\underset{\mathrm{C}_{3} \mathrm{H}_{5}}{\mathrm{CH}_{3}}>\mathrm{CBr}$. $\mathrm{CHBr}$. $\mathrm{CH}_{2} \mathrm{Br}$. 1". $\stackrel{\mathrm{CH}_{3}}{\mathrm{CH}_{2}=\mathrm{C}} \backslash \mathrm{C} \cdot \mathrm{CH}_{--} \mathrm{CH}_{2}$.

$$
\text { 1". } \underset{\mathrm{CH}_{2}=\mathrm{CH}}{\mathrm{CH}_{3}}>\mathrm{C}_{-}^{-}-\mathrm{C}_{-}^{-} \mathrm{CH}_{2} \text {. }
$$

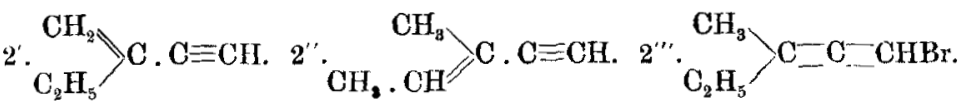

Gelangt man zu $\mathrm{C}_{6} \mathrm{H}_{8}$, d. h. spaltet $\mathrm{C}_{6} \mathrm{H}_{11} \mathrm{Br}_{3}$ drei $\mathrm{BrH}$ $\mathrm{ab}$, und fällt dieses nicht ammoniakalisches Kupferchlorür, so

1) Auf Grund der einmaligen oder zweimaligen Anlagerung von Brom und Abspaltung von Bromwasserstoff. 
ist die Constitution dieses letzteren $1^{\prime}, 1^{\prime \prime}, 1^{\prime \prime \prime}, \mathrm{C}_{6} \mathrm{H}_{10} \mathrm{Br}_{3}$ wird jedenfalls $\mathrm{I}$ sein, und die gesuchte Constitution des $\mathrm{C}_{6} \mathrm{H}_{11} \mathrm{Br}$ wird 1, d. h. $\mathrm{CH}_{3} \cdot \mathrm{CH} \geqslant \mathrm{C}$. $\mathrm{CHBr} . \mathrm{CH}_{3}$ sein. Gewinnt man aber $\mathrm{C}_{6} \mathrm{H}_{8}$, welches ammoniakalisches Kupferchlorür fällt, so ist dieses letztere 2', 2", oder gelangt man nur zu $\mathrm{C}_{8} \mathrm{H}_{9} \mathrm{Br}$ (d. h. spaltet $\mathrm{C}_{6} \mathrm{H}_{11} \mathrm{Br}_{3}$ nur zwei $\mathrm{BrH} \mathrm{ab}$ ), so ist dieses letztere $2^{\prime \prime \prime}$. Jedenfalls aber wird $\mathrm{C}_{6} \mathrm{H}_{11} \mathrm{Br}_{3}$ II sein, und die gesuchte Constitution des $\mathrm{C}_{6} \mathrm{H}_{11} \mathrm{Br}$ wird 2, d. h. ${ }_{\mathrm{C}_{2} \mathrm{H}_{5}}^{\mathrm{CH}_{3}}>\mathrm{CBr}$. $\mathrm{CH}^{-} \mathrm{CH}_{2}$. Wie man sieht, muss unter allen Umständen ${ }^{1}$ ) die Constitution des $\mathrm{C}_{6} \mathrm{H}_{11} \mathrm{Br}$ bestimmt werden können, wenn die Constitution des $\mathrm{C}_{6} \mathrm{H}_{10}$ 2, d. h. ${ }_{\mathrm{CH}_{3} \text {. } \mathrm{CH}}^{\mathrm{CH}_{3}}>\mathrm{C}$. $\mathrm{CH}=\mathrm{CH}_{2}$ ist.

3. Ist $\mathrm{C}_{6} \mathrm{H}_{10}$ von der Constitution 3, d.h. ${ }_{\mathrm{O}_{2} \mathrm{H}_{5}}^{\mathrm{CH}_{3}}>\mathrm{C}=\mathrm{C}-\mathrm{CH}_{2}$, so kann $\mathrm{C}_{6} \mathrm{H}_{11} \mathrm{Br}$

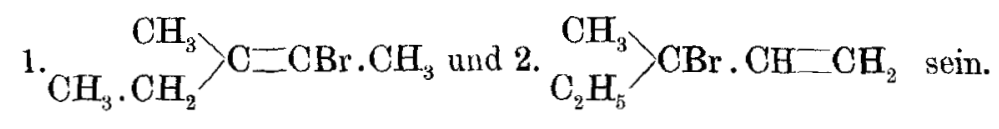

I. $\stackrel{\mathrm{CH}_{3}}{\mathrm{CH}_{2} \mathrm{H}_{5}}>\mathrm{CBr} . \mathrm{CBr}_{2} . \mathrm{CH}_{3}$.

II. $\begin{gathered}\mathrm{CH}_{3} \\ \mathrm{C}_{2} \mathrm{H}_{5}\end{gathered}>\mathrm{CBr}$. $\mathrm{CHBr}$. $\mathrm{CH}_{2} \mathrm{Br}$.

1. $\stackrel{\mathrm{CH}_{2} \mathrm{H}_{5}}{\mathrm{CH}_{2}} \backslash \mathrm{C} . \mathrm{C} \equiv \mathrm{CH}$.

I". $\stackrel{\mathrm{CH}_{3} \cdot \mathrm{CH}_{\mathrm{g}}}{\mathrm{CH}^{\prime}} \mathrm{C} \cdot \mathrm{C} \equiv \mathrm{CH}$.

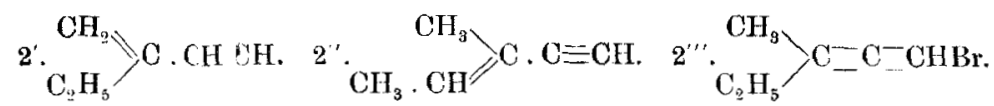

Erhält man $\mathrm{C}_{4} \mathrm{H}_{9} \mathrm{Br}$, d. h. spaltet $\mathrm{C}_{9} \mathrm{H}_{13} \mathrm{Br}_{3}$ nur $2 \mathrm{BrH}$ $a b$, so ist $\mathrm{C}_{6} \mathrm{H}_{3} \mathrm{Br} 2^{\prime \prime \prime}, \mathrm{C}_{6} \mathrm{H}_{11} \mathrm{Br}_{3} \mathrm{II}$, und die gesuchte Constitution des $\mathrm{O}_{i 1} \mathrm{H}_{11} \mathrm{Br}$ ist 2, d. h. ${ }_{\mathrm{C}_{2} \mathrm{H}_{5}}^{\mathrm{CH}_{3}}>\mathrm{CBr} . \mathrm{CH}_{-} \mathrm{CH}_{2}$. Gelangt man dagegen zu $\mathrm{C}_{6} \mathrm{H}_{3}$ (welches ammoniakalisches

1) Auf Grund der einmaligen Anlagerung von Brom und Abspaltung von Bromwasserstoff. 
Kupferchlorür fällen wird), so kann es $1^{\prime}, 1^{\prime \prime}, 2^{\prime}, 2^{\prime \prime}$ sein. Die Constitution des $\mathrm{C}_{6} \mathrm{H}_{11} \mathrm{Br}$ kann in diesem zweiten Falle 1 oder 2 sein, wie früher; wir werden daher in diesem ungünstigen Falle noch die Constitution von $\left(\mathrm{O}_{6} \mathrm{H}_{11} \mathrm{Br} . \mathrm{Br}_{2}-1 \mathrm{BrH}\right)$, $\left(\mathrm{C}_{6} \mathrm{H}_{11} \mathrm{Br} . \mathrm{Br}_{2}-2 \mathrm{BrH}\right)$ untersuchen, d. h. die $\mathrm{zweite}$ Art Reduction anwenden.

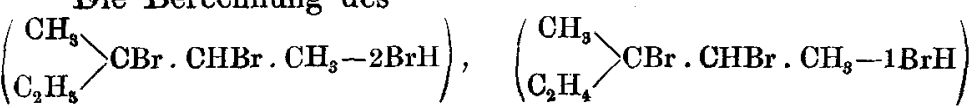

war noch insofern interessant, als sie zeigte, dass auf Grund der Anlagerung von Brom und Abspaltung von Bromwasserstoff wir im Stande sind zu beantworten, in welcher dieser drei Ketten von verschiedener Länge das erste oder das zweite Bromwasserstoffatom sich abspaltet.

Ich habe somit durch die Berechnung einer Reihe beliebig gewählter Beispiele genïgend gezeigt, dass wir, noch ohne zu der zweiten Art der Reduction der Combinationen zu greifen, auf Grund der einmaligen oder zweimaligen Anlagerung von Brom bis zur Sättigung und MaximalAbspaltung von Bromwasserstoff in den überaus meisten Fällen die Constitution von gesättigten und ungesättigten Halogenderivaten, resp. Kohlenwasserstoffen bestimmen können. Ich habe eine Reihe von Beispielen vorgeführt, und bei jedem dieser Beispiele ist gleichzeitig auch die Constitution von höheren Gliedern durch dieselbe Berechnung erkannt worden. Dass es hier, wie überall, günstigere und weniger günstige Fälle giebt, als die vorgeführten, ist selbstrerständlich; eine einfache Berechnung giebt uns aber die Möglichkeit, immer vorauszusehen, welche Schwierigkeiten uns bei der Constitut. 1sbestimmung nach vorliegender Methode erwarten. In den meisten Fällen werden wir im Stande sein, mit rein chemischen Mitteln diese Reduction der Combinationen bis auf eine einzig theoretisch mögliche durchzuführen; in anderen Fällen werden wir zuerst eine theilweise Reduction der Combinationen auf Grund physikalischer Mittel, z. B. der Verbrennungswärme, des Lichtbrechungsvermögens der Körper u. s. w., unternehmen müssen, um dann nach vorliegender Methode die theoretisch einzig mögliche Combination zu finden. Durch die Bestimmung der einzig theoretisch möglichen Combination auf $z w e i$ Wegen, 
492 Hector: Einwirkung von Oxydationsmitteln etc.

wird der Nachweis der Constitution zu einem mathematischsicheren werden.

Am Schlusse möchte ich nur noch darauf aufmerksam machen, dass die Anlagerung von Brom und Abspaltung von Bromwasserstoff die überaus wichtigste und leichteste Darstellungsmethode von gesättigten und ungesättigten Halogenderivaten, resp. Koblenwasserstoffen ist, dass die Darstellung der Körper und die Bestimmung ihrer Constitution somit gleichzeitig geschehen kann, dass bei der Bestimmung der Constitution eines Körpers gleichzeitig die Constitution der meisten dazu dargestellten gesättigten und ungesättigten Verbindungen erkannt wird.

Karlsruhe, 12. Juni 1891.

\section{Einwirkung von 0xydationsmitteln auf aliphatische Thioharnstoffe;}

von

\section{S. Hector.}

In zwei früheren Mittheilungen ${ }^{1}$ ) habe ich meine Untersuchungen über das Verhalten der aromatisch substituirten Thioharnstoffe zu Oxydationsmitteln, besonders zu einer 3 procentigen Wasserstoffsuperoxydlösung, dargelegt. Ich habe nun diese Untersuchungen auf einige Thioharnstoffe der aliphatischen Reihe und zwar den Allyl-, Methyl- und den einfachen Thioharnstoff selbst ausgedehnt. Diese Versuche haben gezeigt, dass die Reaction, die bei der Oxydation der aromatischen Thioharnstoffe mit Wasserstoffsuperoxyd in saurer Lösung vor sich geht, allerdings auch eintritt, wenn aliphatische Thioharnstoffe mit demselben Oxydationsmittel unter übrigens gleichen Bedingungen behandelt werden, aber sich nur zur Hälfte vollzieht. In jenem Falle verläuft die Reaction nach den Gleichungen $(\mathrm{Ar}=$ arom. Radical):

1) Ber. 22, 1176 und 23, 357. 